\title{
Even-odd harmonics generated from above-threshold ionization
}

\author{
Dong-Sheng Guo ${ }^{1,2, \dagger}$, Jing-Tao Zhang ${ }^{3}$, Zhen-Rong Sun ${ }^{1}$, Jin T. Wang ${ }^{2}$, Ju Gao ${ }^{4}$, \\ Zhi-Wei Sun ${ }^{5}$, R. R. Freeman ${ }^{6}$ \\ ${ }^{1}$ State Key Laboratory of Precision Spectroscopy, Department of Physics, East China Normal University, Shanghai 200062, China \\ ${ }^{2}$ Department of Physics, Southern University, Baton Rouge, LA 70813, USA \\ ${ }^{3}$ State Key Laboratory of High Field Laser Physics, Shanghai Institute of Optics and Fine Mechanics, \\ Chinese Academy of Sciences, Shanghai 201800, China \\ ${ }^{4}$ Jingpin Opto-Electronic Co., Jiangsu FOHO Science Park, Fenhu Town, Wujiang 215211, China \\ ${ }^{5}$ Key Laboratory of Microgravity, Institute of Mechanics, Chinese Academy of Sciences, Beijing 100190, China \\ ${ }^{6}$ Department of Physics, Ohio State University, Columbus, OH 43210, USA \\ Corresponding author.E-mail: †dsguophd@gmail.com \\ Received June 14, 2013; accepted August 6, 2013
}

\begin{abstract}
We are reporting a theoretical prediction: The photoelectrons forming above-threshold-ionization (ATI) peaks emit both even and odd harmonics. These harmonics exhibit plateau and cut-off features similar to those odd-only harmonics observed in ATI experiments.
\end{abstract}

Keywords strong-field laser physics, above-threshold inoization, high harmonic generation, evenodd harmonics, nonperturbative quantum electrodynamics

PACS numbers 32.80.Rm, 42.65.Ky, 12.20.Ds, 03.65.Nk

High harmonic generation (HHG) accompanying abovethreshold ionization (ATI) is one of the most interesting phenomena in strong-field laser physics. In the recent two decades, many observations of odd-only harmonics accompanying ATI peaks have been reported $[1,2]$. Almost all related theoretical models are based on an assumption that electrons which emit the odd-only harmonics transit back to the initial bound states [3-9] and are, therefore, called recombination models. Electromagnetic processes conserve charge, parity, and time reversions. The cancelation of even harmonics in theoretical derivations of recombination models is due to the symmetry of initial and final boundary conditions in the sub-Hilbert space of electron, as a natural consequence of the parity conservation or the charge-parity conservation. This success of recombination models really indicates that the electrons emitting odd-only harmonics are not the photoelectrons forming ATI peaks, but those transiting back to the initial state with no contributions to ATI peaks. When more ionized electrons transit back to the initial bound state, the ATI rate should reduce and show stability [10]. Thus, the odd-only HHG is not really accompanying the ATI peaks, rather accompanying the stabilization of atoms in ATI. Then, a natural question is: What kind of harmonics will electrons emit if they do not transit back to the initial state, especially the photoelectrons forming ATI peaks?

Previous theoretical studies proved that the photoelectrons forming ATI peaks had spontaneous emission, enforced by the energy-momentum conservation, and the frequencies of the spontaneous emission were spaced by one laser photon energy [11]. With the asymmetric boundary condition, the harmonics emitted by photoelectrons forming the ATI peaks must show both even and odd orders. Actually, there was already this type of high harmonic spectra observed from plasmas generated by laser light [12-14] and received some attention [1519]. A question is: why ATI experiments do not show the even-odd harmonics? While, this question should not prohibit a theoretical prediction that photoelectrons forming ATI peaks emit both even and odd harmonics. Further studies on the spectra of these harmonics are necessary to facilitate further experimental observations.

In 1991, Kulander et al. [20], in a computer simulation of a three-dimensional hydrogen model, showed evidence of even harmonics. They, using parity conservation, interpreted the evidence as a result of the asymmetry of the electric field. In 1992, Ehlotzky [21] predicted spontaneously emitted high harmonics with both even and odd orders, where the ATI process and the HHG pro- 
cess were assumed to be in parallel. While, these pioneer predictions of even harmonics were neither observed by experimentalists nor discussed enough by theoreticians.

The mechanism of even-odd harmonics discussed here is different from previous ones. In the current discussion, the even-odd harmonic generation follows photoionization in the field. The two processes are in series. This is not from an assumption, rather a derived result from fundamental equations. The even harmonics discussed here is a natural consequence of the asymmetry between the initial and the final electron states due to the parity conservation or charge-parity conservation.

According to Guo, Åberg and Crasemann (GAC) [22], the final state of the photoelectron is not a Volkov state, but a plane wave; the Volkov states play the role of intermediate states. In the single-mode assumption, GAC theory results in an integer condition for the ponderomotive parameter $u_{p} \equiv U_{p} /(\hbar \omega)$, which indicates the existence of a spontaneous light emission [11]. One may think that this is a failure of GAC theory, but we rather think this is a great advantage of using this theory. In contrast with all classical-field treatments, GAC theory has step by step energy and momentum conservation. Only this theory revealed the inadequateness of the single-mode assumption, which was previously adopted by all other theories, because its violates the energy-momentum conservation in the $u_{p}$ non-integer case. The above assertion concerning the final state was soon verified by an existing measurement of the half Kapitza-Dirac effect performed by Bucksbaum et al. [23]. The excellent agreement between Bucksbaum et al.'s measurement [23] and GAC theory in the standing-wave case [24] further strengthened the believe that the extra photon mode has to be included in single-mode photoionization. The theory later was developed with the inclusion of the spontaneously emitted photon mode [11]. The numerical calculations in the current paper are based on this formula. Comparing the current calculations with existing experimental measurements we believe that there are two coexisting mechanisms for HHG, electron recombination and escape. A unified picture of ATI accompanied by HHG is given in the last part of this paper.

The transition formula is the angular distribution for a given ATI peak [11]

$$
\begin{aligned}
& \left.\frac{\mathrm{d}^{4} W}{\mathrm{~d}^{2} \Omega_{\boldsymbol{P}_{f}} \mathrm{~d}^{2} \Omega_{\boldsymbol{k}^{\prime}}}\right|_{j}=\frac{e^{2} \omega^{9 / 2}}{\left(2 m_{e}\right)^{1 / 2}(2 \pi)^{5}}\left(j-e_{b}-u_{p}\right)^{1 / 2} \\
& \quad \times\left(j-u_{p}\right)^{2} \sum_{q}\left(u_{p}-j+q\right)\left|\Phi_{i}(\boldsymbol{P})\right|^{2}\left|\mathcal{X}_{q}\left(\boldsymbol{P}_{f}, \boldsymbol{k}^{\prime}\right)\right|^{2}
\end{aligned}
$$

where $j$ is the transferred-photon number in the ionization process, $\boldsymbol{P}=\boldsymbol{P}_{f}-q \boldsymbol{k}+\boldsymbol{k}^{\prime}, \boldsymbol{P}_{f}$ is the final momentum of the photoelectron; $\Phi_{i}(\boldsymbol{P})$ is the Fourier transform of the initial wavefunction with binding energy $E_{b} \equiv e_{b} \omega$. To add all photoelectrons rates accompanied by different harmonic emissions for a specific ATI peak, the sum in Eq. (1) is performed over all possible integer $q$. The function $\mathcal{X}_{q}\left(\boldsymbol{P}_{f}, \boldsymbol{k}^{\prime}\right)$ is defined by

$$
\begin{aligned}
\mathcal{X}_{q} & \left(\boldsymbol{P}_{f}, \boldsymbol{k}^{\prime}\right) \equiv \frac{1}{\omega} \mathcal{X}_{-j}(z, \eta) \sum_{j^{\prime}} \frac{1}{u_{p}-j^{\prime}} \mathcal{X}_{-j^{\prime}}\left(z_{f}, \eta\right) \\
& \times\left[-\left(\boldsymbol{P}_{f}+\left(j-q-u_{p}\right) \boldsymbol{k}\right) \cdot \boldsymbol{\epsilon}^{\prime *} X_{q-j+j^{\prime}}\left(z_{\boldsymbol{k}^{\prime}}\right)\right. \\
& +e \Lambda \boldsymbol{\epsilon}^{*} \cdot \boldsymbol{\epsilon}^{\prime *} X_{q-j+j^{\prime}+1}\left(z_{\boldsymbol{k}^{\prime}}\right) \\
& \left.+e \Lambda \boldsymbol{\epsilon} \cdot \boldsymbol{\epsilon}^{\prime *} X_{q-j+j^{\prime}-1}\left(z_{\boldsymbol{k}^{\prime}}\right)\right]
\end{aligned}
$$

where $\boldsymbol{k}^{\prime}$ and $\boldsymbol{\epsilon}^{\prime}$ denote the wave vector and the polarization vector of spontaneous emission mode respectively, and $\mathcal{X}_{j}(z, \eta) \equiv \sum_{s=0}^{ \pm \infty} X_{j-2 s}(z) X_{s}(\eta)$ are the generalized phased Bessel functions [25] defined in terms of phased Bessel functions $X_{n}(z)$ of a complex variable, say $z$, and related to the ordinary Bessel functions $J_{n}(r)$ by $X_{n}(z)=J_{n}(r) \exp (\mathrm{i} n \varphi)$, with $z=r \exp (\mathrm{i} \varphi)$. The arguments in Eq. (2) are defined as follows:

$$
\begin{aligned}
& z_{f}=2 \frac{|e| \Lambda}{m_{e} \omega} \boldsymbol{P}_{f} \cdot \boldsymbol{\epsilon}, \quad z_{\boldsymbol{k}^{\prime}}=2 \frac{|e| \Lambda}{m_{e} \omega} \boldsymbol{k}^{\prime} \cdot \boldsymbol{\epsilon} \\
& z=z_{f}+z_{\boldsymbol{k}^{\prime}}, \quad \eta=\frac{1}{2} u_{p} \cos \xi
\end{aligned}
$$

where $\xi$ is the polarization degree, and $\Lambda=$ $|e|^{-1}\left(u_{p} m_{e} \omega\right)^{\frac{1}{2}}$, one half of the classical amplitude of the laser field. In Eq. (2), the transferred-photon number $j$ signifies the order of ATI peaks [11]. The Fourier transform of initial wavefunction $\Phi_{i}(\boldsymbol{P})$ in our numerical calculations is chosen as the one for the outer-most shell $5 P 3 / 2$ of xenon atom with the binding energy $E_{b}=12.1$ $\mathrm{eV}$. The ionization rate for a given ATI order can be obtained by integrating over the solid angles of both the electron and the spontaneous-emission mode; while the total ionization rate is the summation of the rates over all ATI peaks.

Each term in the summation indexed by $q$ in Eq. (1) corresponds to one harmonic, emitted by the electron transiting from the initial bound state to the final plane wave of the $j$ th ATI peak, with the frequency

$$
\omega^{\prime}=\left(q+u_{p}-j\right) \omega
$$

Here $q=n_{i}-n_{f}$ is the overall transferred photon number through the transition. This relation is obtained from the energy conversation required by the scattering theory. The quantity $q+u_{p}-j$ suggests the following physical process: the electron ionizes into the laser field by absorbing $j$ photons, then, exits the field by re-absorbing $q-j$ photons from the field for $q>j$ and converting these 
photons into a high frequency one as the spontaneousemission mode.

This spontaneous emission has a discrete energy spectrum featuring it as harmonics. The other important features shared by this emission and odd-only HHG will be discussed in later context. The spacing between two neighboring harmonics in the spectrum of the spontaneous emission is exactly the laser photon energy $\Delta \omega^{\prime}=$ $\omega \Delta\left[u_{p}-(j-q)\right]=\omega$. The order of a harmonic can be determined by $q-j+\left[u_{p}\right]$. It is shown again that the ponderomotive energy, as a component of the ponderomotive four momentum [26], cannot be kept by the photoelectron when it exits the field; but turns into, as a part, the energy of the light harmonic.

To obtain the angular distribution formula for the spontaneous emission in a given order, we start from Eq. (1) with the following steps. i) We only calculate one term in Eq. (1) without summing over $q$, because $q$ specifies one harmonic. ii) We integrate over the solid angle in the electron momentum space because photoelectrons escaping in different directions may generate the same harmonic. iii) We sum over all different ATI peaks because photoelectrons forming different ATI peaks may generate the same harmonic. Thus, we have the angular distribution formula for the spontaneous emission

$$
\begin{gathered}
\left.\frac{\mathrm{d}^{2} W}{\mathrm{~d}^{2} \Omega_{\boldsymbol{k}^{\prime}}}\right|_{q}=\frac{e^{2} \omega^{9 / 2}}{\left(2 m_{e}\right)^{1 / 2}(2 \pi)^{5}} \sum_{j}\left(j-e_{b}-u_{p}\right)^{1 / 2}\left(j-u_{p}\right)^{2} \\
\times\left(u_{p}-j+q\right) \int \mathrm{d}^{2} \Omega_{\boldsymbol{P}_{f}}\left|\Phi_{i}(\boldsymbol{P})\right|^{2}\left|\mathcal{X}_{q}\left(\boldsymbol{P}_{f}, \boldsymbol{k}^{\prime}\right)\right|^{2}
\end{gathered}
$$

where the summation over $j$ corresponds to adding all the contributions from photoelectrons forming different ATI peaks. The total yield of a harmonic is obtained by integrating over the solid angle of $\boldsymbol{k}^{\prime}$, the propagation vector of the harmonic mode, in Eq. (5).

In our sample calculation, the wavelength of the incident laser is chosen as $1064 \mathrm{~nm}$ and linearly polarized. The laser intensity varies from $10^{13} \mathrm{~W} / \mathrm{cm}^{2}$ to $3 \times 10^{14}$ $\mathrm{W} / \mathrm{cm}^{2}$. Our calculations include angular distributions and spectral distributions of the spontaneous emission. The angular distribution obtained reveals a pronounced forward-propagation property, as well as the backwardpropagation property. Here the forward direction means the laser propagation direction. The harmonic emission rate in the forward direction is, at least, twice that of the emission in the laser polarization plane, as shown in Fig. 1. The spectral distribution further reveals that the spontaneous emission does have all main characters of the experimentally observed HHG in the odd-only case, such as the plateau and cutoff structures, as shown in Figs. 2 and 3.

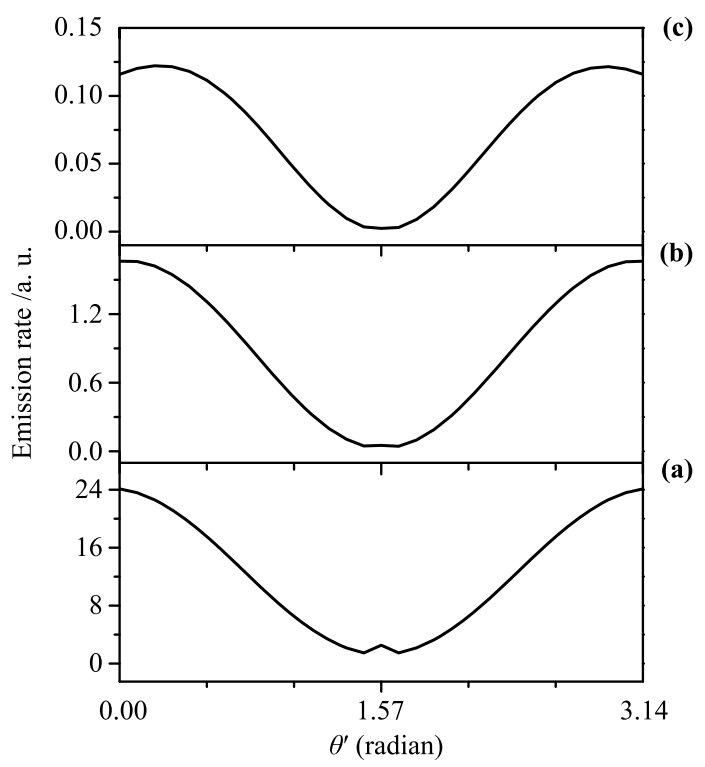

Fig. 1 Angular distributions of several harmonics: (a) 2nd; (b) 20th; and (c) 40th. The laser intensity is chosen as $10^{14} \mathrm{~W} / \mathrm{cm}^{2}$, and the azimuthal angle is $\phi^{\prime}=0$.

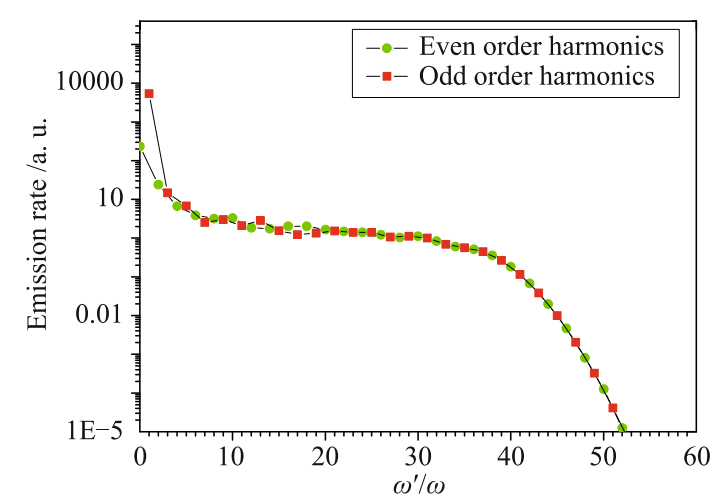

Fig. 2 The calculated HHG spectrum along the laser propagation for laser intensity $1 \times 10^{14} \mathrm{~W} / \mathrm{cm}^{2}$. Other conditions are the same as those in Fig. 1. Other parameters: $E_{b} / \omega=10.4, \xi=0, \lambda=1064$ $\mathrm{nm}$. Line with dot-centered circles denotes the spectrum of the even harmonics and line with squares denotes the spectrum of the odd harmonics.

From the experimental points of view, high harmonics have the forward-propagation property [27]. Most earlier calculations assumed that the high harmonics propagate in this direction. From the theoretical points of view, the forward propagation assumption needs to be removed from the theory. To fulfill this purpose, we calculate the angular distributions of several harmonics. Figure 1 shows the angular distributions of several harmonics at laser intensity $10^{14} \mathrm{~W} / \mathrm{cm}^{2}$. Even though our formula allows us to calculate the full angular distribution of all harmonics, to make the problem simple, we only calculate the angular distribution in the laser polarizationpropagation plane (the $x-z$ plane) which is a function of the scattering angle $\theta^{\prime}$ for the fixed azimuthal angle 


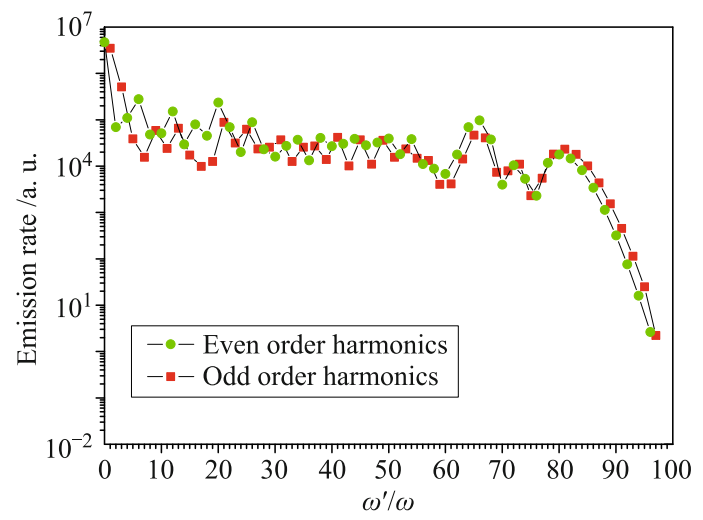

Fig. 3 The calculated HHG spectrum along the laser propagation for laser intensity $2.5 \times 10^{14} \mathrm{~W} / \mathrm{cm}^{2}$. Other conditions are the same as those in Fig. 2.

$\phi^{\prime}=0$. Figure $1(\mathrm{a})$ is the angular distribution of the second harmonic. The emission rate in the forward direction $\theta^{\prime}=0$, as well as the backward direction $\theta^{\prime}=\pi$, are much larger than those in the transverse direction, $\theta^{\prime}=0.5 \pi$. For the harmonics of higher orders, the maximum of the emission rate is still in the forward direction and the backward direction, as shown in Fig. 1(b) for the 20th order harmonic. For further higher-order ones, even though the maximum of the emission rate slightly departs from the forward direction, as shown in Fig. 1(c), the forward-propagation preference still generally holds.

Since the harmonics emitted are mostly in the forward and backward directions, we set $\theta^{\prime}=0$ in the calculations of the spectral distribution of harmonics. Our calculations show that the emission rate falls off drastically after few beginning orders, with increasing of the harmonic order, then exhibits a plateau where all the harmonics have the comparable strength, and finally ends up with a sharp cutoff. The plateau and cutoff, two outstanding features of the spectra of odd-only harmonics, are now shared with the spectra of even-odd harmonics as a consequence of our calculations.

Just as in the odd-only case, the plateau and the cutoff frequency of even-odd harmonics also vary with the laser intensity. At low laser intensities, the plateau and the cutoff are both not outstanding. With increasing of laser intensity, a clear plateau and a sharp cutoff appear in the harmonic spectra, as shown in Figs. 2 and 3 at laser intensities $10^{14} \mathrm{~W} / \mathrm{cm}^{2}$ and $2.5 \times 10^{14} \mathrm{~W} / \mathrm{cm}^{2}$, respectively. Because the even harmonics produced in the photoelectron-exit process have a comparable strength with the odd ones, we term the harmonics in the current discussion as even-odd harmonics.

From our calculations, we assert that there are two coexisting mechanisms to produce high harmonics. In the recombination mechanism, photoelectrons fall back to the original atomic bound state with an emission of odd-only harmonics. In the escape mechanism, photoelectrons escape from the laser field with an emission of both even and odd harmonics.

The next question is: How to observe these even harmonics? With the two mechanisms of HHG, let us think about two extreme cases. Case I: The atoms in the laser field show stability and the ionization rate is rather low. In this case, most electrons are recombined, the HHG spectra will show odd-only. Since there is no much even harmonics, if the other sources of noises can be wellcontrolled, one should observe clean odd-only harmonics with good ratio of signal versus noise. Case II: The atoms do not show stability in the laser field and the ionization rate is high. In this case, most electrons escape from the laser field to form ATI peaks. The electrons forming ATI peaks emit both even and odd harmonics. Since there are no many recombined electrons which emit oddonly harmonics, if one still observes "odd-only" spectra, these spectra cannot be very clean, i.e., must have big noise. The even harmonics must hide in the noise. With improved experimental techniques, it is very possible to separate the even harmonics from those high noises.

In closing this paper, we want to emphasize that the emission of even-odd harmonics from ATI process is an inevitable theoretical result. In spite of detailed theoretical proof, a quick way to look at this result is using the Furry' theorem developed in the early years of quantum electrodynamics [28]. When Furry theorem applies to a nonvanishing Feynman diagram with a closed electron line, the number of the total external photon lines in the diagram is an even number. The closed electron line means that the electron transits back to its original state. The even-number requirement for the external photon lines forces the harmonics emitted by the electron to be an odd order. The electrons which do not transit back to its original state, such as photoelectrons produced in ATI, must emit both even and odd harmonics. The assertion of the even-odd harmonics from ATI electrons is a natural consequence of fundamental theories in physics, also supports the widely used recombination models as their supplemental. The history of the observation of HHG from ATI experiments is still very young, during which there are not enough emphasized theoretical predictions of even-odd HHG. Many important theoretical predictions in physics took much longer time than this short history, such as gravitational waves which has been predicted nearly a century still without direct evidences. We believe that with our emphasized prediction, the even-odd harmonics will be observed in the near future after more efforts devoted by both experimentalists and theoreticians. 
Acknowledgements This work was supported in part by the National Natural Science Foundation of China (Grant Nos. 11174304 and 61078080), the National Basic Research Program of China (973 Program) (Grant Nos. 2010CB923203 and 2011CB808103), and also the National Natural Science Foundation of China under Grant No. 11172302

\section{References}

1. A. Mcpherson, G. Gibson, H. Jara, U. Johann, T. S. Luk, I. A. McIntyre, K. Boyer, and C. K. Rhodes, Studies of multiphoton production of vacuum-ultraviolet radiation in the rare gases, J. Opt. Soc. Am. B, 1987, 4(4): 595

2. X. F. Li, A. L'Huillier, M. Ferray, L. A. Lompré, and G. Mainfray, Multiple-harmonic generation in rare gases at high laser intensity, Phys. Rev. A, 1989, 39(11): 5751

3. J. Gao, F. Shen, and J. G. Eden, Quantum electrodynamic treatment of harmonic generation in intense optical fields, Phys. Rev. Lett., 1998, 81(9): 1833

4. X. M. Tong and S. I. Chu, Generation of circularly polarized multiple high-order harmonic emission from two-color crossed laser beams, Phys. Rev. A, 1998, 58(4): R2656

5. L. Gao, X. Li, P. Fu, R. R. Freeman, and D.-S. Guo, Nonperturbative quantum electrodynamics theory of high-order harmonic generation, Phys. Rev. A, 2000, 61(6): 063407

6. P. Antoine, A. L'Huillier, and M. Lewenstein, Attosecond pulse trains using high-order harmonics, Phys. Rev. Lett., 1996, 77(7): 1234

7. I. P. Christov, M. M. Murnane, and H. C. Kapteyn, Highharmonic generation of attosecond pulses in the "singlecycle" regime, Phys. Rev. Lett., 1997, 78(7): 1251

8. J. Zhou, J. Peatross, M. M. Murnane, H. C. Kapteyn, and I. P. Christov, Enhanced high-harmonic generation using 25 fs laser pulses, Phys. Rev. Lett., 1996, 76(5): 752

9. M. Lewenstein, P. Balcou, M. Y. Ivanov, A. L'Huillier, and P. B. Corkum, Theory of high-harmonic generation by lowfrequency laser fields, Phys. Rev. A, 1994, 49(3): 2117

10. S. I. Chu, Laser-induced stabilization in atomic and molecular-systems, J. Chinese Chem. Soc., 1995, 42(2): 325

11. J. Gao, D. S. Guo, and Y. S. Wu, Resonant above-threshold ionization at quantized laser intensities, Phys. Rev. A, 2000, 61(4): 043406

12. R. L. Carman, D. W. Forslund, and J. M. Kindel, Visible harmonic emission as a way of measuring profile steepening, Phys. Rev. Lett., 1981, 46(1): 29

13. R. L. Carman, C. K. Rhodes, and R. F. Benjamin, Observation of harmonics in the visible and ultraviolet created in $\mathrm{CO}_{2}$-laser-produced plasmas, Phys. Rev. A, 1981, 24(5): 2649
14. F. Quéré, C. Thaury, P. Monot, S. Dobosz, Ph. Martin, J. P. Geindre, and P. Audebert, Coherent wake emission of highorder harmonics from overdense plasmas, Phys. Rev. Lett., 2006, 96(12): 125004

15. Y. I. Salamin and F. H. M. Faisal, Harmonic generation by scattering circularly polarized light of arbitrary intensity from free electrons of arbitrary initial velocity, Phys. Rev. A, 1997, 55(5): 3964

16. Y. I. Salamin and F. H. M. Faisal, Harmonic generation by superintense light scattering from relativistic electrons, Phys. Rev. A, 1996, 54(5): 4383

17. C. Bamber, K. T. McDonald, E. J. Prebys, C. Bamber, S. Boege, T. Kotseroglou, A. C. Melissinos, D. D. Meyerhofer, W. Ragg, D. L. Burke, R. C. Field, G. Horton-Smith, A. C. Odian, J. E. Spencer, D. Walz, S. C. Berridge, W. M. Bugg, K. Shmakov, and A. W. Weidemann, Observation of nonlinear effects in Compton scattering, Phys. Rev. Lett., 1996, 76(17): 3116

18. D. G. Lappas, M. V. Fedorov, and J. H. Eberly, Spectrum of light scattered by a strongly driven atom, Phys. Rev. A, 1993, 47(2): 1327

19. E. Esarey, S. K. Ride, and P. Sprangle, Nonlinear Thomson scattering of intense laser pulses from beams and plasmas, Phys. Rev. E, 1993, 48(4): 3003

20. K. C. Kulander, K. J. Schafer, and J. L. Krause, Dynamic stabilization of hydrogen in an intense, high-frequency, pulsed laser field, Phys. Rev. Lett., 1991, 66(20): 2601

21. F. Ehlotzky, Laser-induced Compton scattering from a bound electron, Can. J. Phys., 1992, 70(1): 72

22. D. S. Guo, T. Åberg, and B. Crasemann, Scattering theory of multiphoton ionization in strong fields, Phys. Rev. A, 1989, 40(9): 4997

23. P. H. Bucksbaum, D. W. Schumacher, and M. Bashkansky, High-intensity Kapitza-Dirac effect, Phys. Rev. Lett., 1988, 61(10): 1182

24. D. S. Guo and G. W. F. Drake, Multiphoton ionization in circularly polarized standing waves, Phys. Rev. A, 1992, 45(9): 6622

25. X. Hu, H. Wang, and D. S. Guo, Phased Bessel functions, Can. J. Phys., 2008, 86(7): 863

26. J. Gao, D. Bagayoko, and D. S. Guo, The ponderomotive four-momentum, Can. J. Phys., 1998, 76(1): 87

27. J. W. G. Tisch, R. A. Smith, J. E. Muffett, M. Ciarrocca, J. P. Marangos, and M. H. R. Hutchinson, Angularly resolved high-order harmonic generation in helium, Phys. Rev. A, 1994, 49(1): R28

28. W. H. Furry, A symmetry theorem in the positron theory, Phys. Rev., 1937, 51(2): 125 